\title{
L'anti-journal de voyage en Amérique de Miron Białoszewski (AAmérique, 1988 et Journal secret, 2012)
}

\author{
Alexander FiuT \\ Université Jagellonne, Cracovie \\ Traduit du polonais par Iwona VIGOT
}

Le voyage en Amérique est un défi pour tout Européen. Ce défi est encore plus grand pour un poète du bloc soviétique qui traverse l'Océan à un moment particulièrement défavorable pour son pays. Le poète polonais Miron Białoszewski (1922-1983) a entrepris son voyage au moment où la Pologne socialiste se trouvait encore en «état de siège », un état de siège proclamé par le général Jaruzelski en décembre 1981, dans le but d'étouffer le syndicat indépendant Solidarność [Solidarité]. Białoszewski a laissé deux témoignages de son séjour aux États-Unis : le premier est un récit de voyage intitulé $A$ Ameryka [AAmérique], dont le processus de publication a été interrompu par la mort de l'auteur et qui est paru seulement cinq années après celle-ci, en 1988 ; le deuxième est constitué par une partie de son Tajny dziennik [Journal secret], qui n'a été publié qu'en 2012. Le Journal secret nous donne des indications précises sur les dates du séjour de Białoszewski aux États-Unis, à savoir du 11 octobre au 23 novembre 1982.

La confrontation de ces deux récits nous amène à la question des limites de la sphère privée et à celle de l'autobiographisme dans la littérature. Dans le cas de Białoszewski, cette question est particulièrement complexe car toutes ses œuvres en prose et toute sa poésie peuvent être lues comme des formes autobiographiques, comme une sorte de journal intime de l'auteur. Dans toute son œuvre, Białoszewski, en gommant intentionnellement la frontière entre le héros fictif et lui-même, puise 
SLOVO

190 Les Voyages lointains des écrivains polonais $\left(\mathrm{XX}^{\mathrm{e}}-\mathrm{XXI}^{\mathrm{e}}\right.$ siècles $)-\mathrm{n}^{\circ} 51$

dans son expérience et élève la banalité du quotidien au rang d'œuvre d'art. Le Journal secret, tenu systématiquement par l'écrivain du 20 novembre 1975 jusqu'à sa mort, contient des notes prises à chaud, une véritable matière première, qui sera par la suite retravaillée dans les autres textes. Ajoutons que Białoszewski a donné à plusieurs de ses œuvres la forme du journal intime. Dans le cas d'AAmérique, en revanche, il a plutôt écrit un « anti-journal ». Pour quelles raisons ?

La nécessité de supprimer certains extraits pour ne pas se heurter à la censure a certainement été une des causes de l'abandon de la forme du journal intime. Une répugnance à dévoiler sa vie intime, le désir de mettre en valeur son attitude apolitique, la loyauté envers ses amis en exil et la crainte que les «services spéciaux » de la Pologne populaire ne s'intéressent de trop près à ces derniers : voilà les principales raisons pour lesquelles l'écrivain n'a pas relaté dans son Journal secret ses rencontres dans le milieu gay, ses expériences homosexuelles, ses conversations téléphoniques avec le poète polonais Czesław Miłosz ${ }^{1}$, ni les scènes des manifestations de la diaspora polonaise devant le consulat de la République populaire de Pologne. D’un côté, Białoszewski ne voulait pas révéler ses contacts avec les émigrés polonais, et de l'autre, il savait que certains passages de sa prose, trop transgressifs sur le plan des mœurs, seraient rejetées par la censure.

Le titre, AAmérique, propose une autre réponse à la question de savoir pourquoi Białoszewski a écrit un «anti-journal ». C'est la dénomination d'une ligne du métro newyorkais qui a donné à Białoszewski l'idée du titre : «ça m’évoque l'Amérique. Deux “A”, c'est comme s'il y avait un grand point d'exclamation ${ }^{2} \gg$. Que signifie le point d'exclamation ? L'admiration ? L'étonnement ? L'épouvante? L'impuissance ? Qui parle ici ? L'auteur ? Un héros qui lui ressemble ? Białoszewski s'efforce de comprendre l'Amérique en y observant la vie quotidienne, en évitant soigneusement de généraliser à la hâte. En même temps - comme c'est le cas dans toute son œuvre - il souhaite habiller ses impressions dans une forme langagière originale et issue du langage courant. En suggérant que le héros-narrateur d'AAmérique est dans une large mesure son propre alter ego, il ne rompt pas seulement avec la tradition du journal intime, dans laquelle s' inscrit le Journal secret. Il désarticule le « je » du journal intime et entreprend un jeu retors avec plusieurs rôles : celui d'écrivain polonais à l'étranger, d'Européen dans le Nouveau Monde, de provincial au centre de la civilisation.

1. Czesław Miłosz (1911-2004), prix Nobel de la littérature en 1980, a demandé l'asile politique à la France en 1951 et s'est installé aux États-Unis en 1960.

2. Kojarzy mi się to z Ameryką. Dwa "A" to jakby z wielkim wykrzyknikiem, BIA£oszewsKI, 2000, p. 222. Les traductions des passages de Białoszewski sont d'Iwona Vigot. 
En tant qu'écrivain de la République populaire de Pologne à qui les autorités ont remis un passeport et aimablement accordé la permission de quitter le pays afin de recevoir un prix littéraire aux États-Unis, il se comporte de manière véritablement scandaleuse. Non seulement il ne représente pas dignement la société socialiste et la culture polonaise, ce que le régime socialiste attend pourtant de lui, mais il se compromet ostensiblement et recherche la provocation. Il évite à tout prix d'être mêlé aux discours sur la Pologne socialiste. Il n'est pas intéressé par ce qui se passe dans son pays. Il déclare ouvertement : « Je me garde de "l'empolonisation". Je veux l'Amérique en Amérique ${ }^{3}$. » En écrivant ces mots, il exprime sa répugnance envers toute forme de récupération politique et sa volonté de se détacher des stéréotypes polonais ou européens au sujet du Nouveau Monde, tout en manifestant son désir de se rapprocher de la conscience d'un Américain moyen. Il se rend, certes, à la remise du prix qui lui a été décerné, rencontre des lecteurs, récite et lit son œuvre en public, mais il ne cache pas qu'il préfère traîner dans les rues de New York, entrer dans des sex-shops, acheter des photos pornographiques, fréquenter des cinémas pornographiques et observer les comportements érotiques des Américains. Avec son humour sarcastique, il décrit des scènes à caractère sexuel avec maints détails. Dans AAmérique on trouve une version nettement édulcorée des descriptions présentes dans le Journal secret, où l'on trouve des récits détaillés d'orgies sexuelles dans les cinémas pornos pour gays.

On pourrait s'attendre à ce qu'un habitant d'Europe centrale soit ébloui et écrasé par la grandeur, la richesse et la dynamique de New York. Il n'en est rien ! Si Białoszewski s'intéresse à l'histoire de la ville et se rend parfois dans les musées de New York, il y remarque surtout des ressemblances avec certaines villes européennes. En arrivant à l'hôtel, il note : «La circulation dans la ville n'est pas aussi intense que ce à quoi je m'attendais [...]. Les maisons sont hautes mais le silence et le vide règnent. Comme à Przysnysz ${ }^{4} \gg$, une petite ville de la province polonaise. Il remarque des trottoirs bancals, des piétons qui traversent la rue au feu rouge, des poubelles devant des maisons. Les étals des marchés et des rues lui rappellent le Varsovie d'avant la guerre. Le « faux ${ }^{5} \gg$ gothique de la cathédrale Saint-Patrick l'intrigue. D’après lui, New York est, en fin de compte, « démodé, souvent négligé, du moins la majeure partie de la ville, avec ses bâtiments en forme de parts d'un gigantesque gâteau rond, ses monuments en fer, ses immeubles, ses cages d'escaliers

3. Bronię sięprzed zapolaczeniem. Chcę Ameryki w Ameryce, BiAŁoszewski, 2000, p. 229. 4. Ruch nie taki szalony, jak sie spodziewatem [...] Domy wielkie, ale cicho i pusto. Jak w Przasnyszu, Biazoszewski, 2000, p. 220.

5.Podrabiany, BiaŁoszewski, 2000, p. 224. 
SLOVO

192 Les Voyages lointains des écrivains polonais $\left(\mathrm{XX}^{\mathrm{e}}-\mathrm{XXI}^{\mathrm{e}}\right.$ siècles $)-\mathrm{n}^{\circ} 51$

en bois ${ }^{6} \gg$. Par son plan géométrique, New York rappelle également une ville médiévale, avec un parc en son centre, en guise de place du marché. Białoszewski reste totalement indifférent au consumérisme. Ses besoins matériels sont modestes mais il s'étonne de constater que le service dans les magasins est assez lent, tout comme en Pologne. Il note : « J'achète du raisin, des tomates, des mandarines. Tout y est si beau qu'on s'y ennuie ${ }^{7}$. » Il venait pourtant d'un pays où les marchandises, même de première nécessité, manquaient continuellement, et où les queues devant les magasins d'alimentation étaient un spectacle ordinaire !

C'est d'une province lointaine que Białoszewski est venu dans la métropole mondiale. Mais il n'a pas de complexes; il s'y sent comme chez lui et ne cesse de le souligner. Il avoue : «J'aime New York, et plus précisément Manhattan. Ambiance de grande ville, on s'y sent comme chez soi. Le grand monde ${ }^{8}$. $\gg$ Les gratte-ciel ne l'impressionnent guère car, écrit-il : «Il ne faut pas se laisser aller au vertige. On peut s'y habituer'. » Sa mauvaise connaissance de l'anglais ne l'empêche pas d'apprendre rapidement à circuler dans le centre de la ville et d'emprunter le métro. Il visite différents quartiers. New York peut-elle être dangereuse ? Oui, comme toutes les grandes villes, écrit Białoszewski, et il raconte qu'il a déjà été roué de coups, un soir, dans le centre de Varsovie. Comme toujours, c'est l'extraordinaire diversité humaine qui l'attire le plus: diversité ethnique, coupes de cheveux, vêtements, coutumes et comportements - un véritable théâtre vivant, scintillant, spontané, objet d'une métamorphose permanente. Ainsi, dans le quartier chinois, son regard est attiré par « les vieilles grosses Chinoises qui marchent comme des momies d'un magasin à l'autre ${ }^{10} \gg$; dans le quartier juif, par un petit garçon aux papillotes tressées; dans une des rues principales de Manhattan, par une femme noire qui interpelle les passants en criant « Jésus t'attend ${ }^{11} ! »$.

6. Staroświecki, dosyć zaniedbany, przynajmniej $w$ dużej części, $z$ tradycyjnymi tortowymi narożnikami, z pomnikami z żelaza, $z$ kamienicami, z drewnianymi klatkami schodowymi, Biatoszewski, 2000, p. 228.

7.Kupuje winogrona, pomidory, mandarynki. Wszystko tu jest dorodne. Aż do nudności, BiaŁoszewski, 2000, p. 223.

8.Podoba mi sie Nowy Jork. To znaczy Manhattan. Wielkomiejsko i swojsko. Światowo, BiaŁoszewski, 2000, p. 223.

9. Nie trzeba ulegać sensacjom wysokości. Do tego można się przyzwyczaić, BIAŁOsZEWsKI 2000, p. 224.

10. Stare grube Chinki chodzace mumiowatym krokiem od sklepu do sklepu, BIA£OSZEWSKI, 2000, p. 227.

11.Jezus czeka na ciebie!, BiAŁoszewski, 2000, p. 222. 
En quoi consiste la particularité de l'«anti-journal» de voyage de Miron Białoszewski ? Pour mieux l'appréhender, il est intéressant de recourir à l'image du Nouveau Monde qui se dégage d'un autre « anti-journal », Amérique, de Jean Baudrillard, publié en 1986.

Les deux auteurs sont incontestablement fascinés par les rues de New York. C'est là, et non dans les musées, qu'ils recherchent la vérité sur l'Amérique. Le poète polonais concentre son attention sur les individus, les petites scènes de la vie ordinaire, les comportements des représentants des différentes ethnies et cultures. Le sociologue français, lui, utilise la notion de communauté, visant à la fois la définition et la synthèse. Il écrit par exemple :

On dit : en Europe la rue est vivante, en Amérique elle est morte. C'est faux. Rien de plus intense, de plus électrisant, de plus vital et de plus mouvementé que les rues de New York. [...] Des millions de gens l'occupent, errants, nonchalants, violents, comme s'ils n'avaient rien d'autre à faire, et sans doute n'ont-ils réellement rien d'autre à faire que de produire le scénario permanent de la ville. (Baudrillard, 1986, p. 43)

On voit ici que Baudrillard comme Białoszewski s’interrogent sur la théâtralité des comportements des New-Yorkais, qui donne à leur façon d'être un caractère inquiétant: «New York se donne, par une complicité merveilleuse de toute la population, la comédie de sa catastrophe [...]»(Baudrillard, 1986, p. 49). Mais lorsqu'il compare l'Amérique à l'Europe, Baudrillard recherche plutôt des différences que des ressemblances. Il note ainsi que le trafic new-yorkais est « intense, relativement véhément et silencieux (ce n'est pas le trafic nerveux et théâtral à l'italienne) » (Baudrillard, 1986, p. 43).

Les deux auteurs sont intrigués par la beauté des « races sombres ». Baudrillard trouve que « le pigment des races sombres [...] » crée « une beauté - non sexuelle : animale et sublime - qui manque désespérément aux visages blêmes » (Baudrillard, 1986, p. 40). Białoszewski, quant à lui, est frappé par autre chose. Il constate que «la beauté des blancs impressionne souvent. Toutefois - objectivement, d'un point de vue dansant et théâtral - c'est une race peu rythmique, lourde, avachie, prétentieuse, paresseuse, maniaque, ne résistant pas à la concurrence dans sa façon d'être extérieure, envisagée comme art, dans la beauté animale ${ }^{12} \gg$.

12. Uroda biatych często dziata. Ale - biorac bezstronnie a tanecznie, teatralnie - to rasa mato rytmiczna, cięzka, krowiasta, z pretensja, leniwa, a porzadnicka, nie wytrzymująca konkurencji w zewnętrzności bycia, jako sztuki, w urodzie zwierzęcia, BIAŁOSZEWSKI, 2012, p. 803-804. 
SLOVO

194 Les Voyages lointains des écrivains polonais $\left(\mathrm{XX}^{\mathrm{e}}-\mathrm{XXI}^{\mathrm{e}}\right.$ siècles $)-\mathrm{n}^{\circ} 51$

Lorsque Białoszewski contemple New York du haut d'une des tours du World Trade Center, «les façades de verre modernes des gratte-ciels » lui rappellent « de pauvres crabes par leurs dimensions et leur apparence ${ }^{13} »$. Baudrillard, au contraire, y voit le symbole de la postmodernité de l'Amérique. Il fait ici un parallèle intéressant :

Face aux downtowns et aux ensembles de gratte-ciel américains, la Défense perd le bénéfice architectural de la verticalité et de la démesure pour avoir enserré ses buildings dans une scène à l'italienne, dans un théâtre fermé circonscrit par un boulevard périphérique. Jardin à la française en quelque sorte : un bouquet de buildings avec un ruban autour. (Baudrilllard, 1986, p. 42)

«Un bouquet de buildings » ? Białoszewski compare les maisons cossues entourant Central Park à un lieu de stockage de meubles gigantesques : «Comme si on avait posé des buffets les uns à côté des autres ${ }^{14}$. » D'après Baudrillard, « l'anti-architecture », « la sauvage, l'inhumaine, celle qui dépasse l'homme, elle s'est faite ici toute seule, à New York, et sans considération de niche, de bien-être ou d'écologie idéale [...] elle a parié sur le ciel et sur l'enfer » (Baudrillard, 1986, p. 42). Selon Białoszewski, les anti-villes américaines sont des rangées infinies de petites maisons, ennuyeuses de par leur ressemblance : «En Amérique, il y a beaucoup d'anti-villes. New York: une montagne de verre (Manhattan) entourée d'eau, et au-delà de cette eau, d'une autre anti-ville ${ }^{15}$. »

Il est frappant que Białoszewski semble ne pas avoir remarqué l'immensité de l'espace du continent américain. La nature l'ennuie visiblement. Cependant, pour Baudrillard, la nature américaine, étourdissante par son immensité, indomptée, primordiale, est la clé du mystère de la civilisation américaine, tendue vers le passé et vers l'avenir, mais surtout annonciatrice d'une catastrophe. Sa vision de l'Amérique est imprégnée de l'image du désert et synonyme du manque de sens, non seulement de l'existence humaine sur terre mais aussi de toute la culture : «L'inhumanité de notre monde futur, asocial et superficiel, trouve d'emblée ici sa forme esthétique et sa forme extatique. Car le désert n'est que cela : une critique extatique de la culture, une forme extatique de la disparition » (Baudrillard, 1986, p. 16/17).

13. Nowoczesne szyby drapaczy przypominaty skala i rodzajem biedne kraby, BIAŁOSZEWSKI, 2012, p. 811.

14. Tak jakby stat kredens przy kredensie, BIA£OsZEWSKI, 2000, p. 229.

15.W Ameryce wiele jest antymiast. New York - szklana góra (Manhattan) otoczona woda, a za woda antymiastem, BIAŁOSZEWSKI, 2012, p. 817. 
Quelles conclusions peut-on tirer de ce parallèle rapide ? On voit clairement que Baudrillard apparaît dans son « anti-journal » dans trois rôles principaux : celui du sociologue, celui du Français et celui de l'Européen. Toutefois, contrairement à Białoszewski, il ne remet aucun rôle en question et les considère tous comme complémentaires. Białoszewski explore d'un côté les affinités entre l'Amérique et l'Europe, et de l'autre, il rend ordinaire ce qui est extraordinaire. Baudrillard, au contraire, souligne l'étrangeté du Nouveau Monde et rend ainsi extraordinaire grâce à la notion de simulacre - ce qui y apparaît d'abord comme ordinaire. Il est bien sûr compréhensible que le poète soit avant tout fasciné par le détail isolé, l'expressivité des personnes et des situations dans lesquelles celles-ci sont engagées. En revanche, le sociologue et anthropologue est incité, par l'observation, à établir des diagnostics afin de conférer aux phénomènes culturels et sociaux qu'il remarque la dimension d'une synthèse sur la civilisation américaine. Toutefois, il est possible d'approfondir une telle explication.

Białoszewski contemple l'Amérique d'un regard, peut-être non dénué de tout a priori, mais du moins façonné par une autre expérience historique, une vision du monde originale et poétique. Baudrillard trouve surtout dans ce voyage américain la confirmation de ses diagnostics catastrophistes. D'où une de ses brillantes formules :

L'Amérique n'est ni un rêve, ni une réalité, c'est une hyperréalité. C'est une hyperréalité parce que c'est une utopie qui dès le début s'est vécue comme réalisée. [...] Il se peut que la vérité de l'Amérique ne puisse apparaître qu'à un Européen, puisque lui seul trouve ici le simulacre parfait, celui de l'immanence et de la transcription matérielle de toutes les valeurs. Les Américains, eux, n'ont aucun sens de la simulation. Ils en sont la configuration parfaite, mais ils n'en ont pas le langage, étant eux-mêmes le modèle. (Baudrillard, 1986, p. 91)

Même s'il n'est pas un Américain, Białoszewski n’éprouve pas cette impression de simulation. Le monde contemporain n'est pas pour lui, contrairement à Baudrillard, une circulation de signes vides dans un espace privé de sens. Il se réapproprie le mythe américain et le transforme à sa façon. Il ne trouve pas que la culture européenne soit une culture de l'épuisement et synonyme de décadence. $\mathrm{Au}$ contraire, elle reste toujours pour lui un soutien et un environnement naturel. En se débarrassant des rôles imposés, il essaie d'observer l'Amérique en adoptant le point de vue qui lui est le plus proche, souverain, au-delà des frontières et des ethnies, tout simplement interhumain, discrètement compatissant, plein d'humour et d'indulgence. 
SLOVO

196 Les Voyages lointains des écrivains polonais $\left(\mathrm{XX}^{\mathrm{e}}-\mathrm{XXI}^{\mathrm{e}}\right.$ siècles $)-\mathrm{n}^{\circ} 51$

\section{Bibliographie}

Baudrillard Jean, 1986, Amérique, Grasset, Paris, 252 p.

Białoszewski Miron, 2000 [1988], AAmeryka [AAmérique] in Mate i większe prozy opublikowane po roku 1980 [Petites et plus grandes proses publiées après 1980], PIW, Varsovie.

Białoszewski Miron, 2012, Tajny dziennik [Journal secret], Znak, Cracovie, $920 \mathrm{p}$.

Miron Białoszewski (1922-1983), poète, prosateur et dramaturge, a laissé deux témoignages de son séjour aux États-Unis, effectué à l'automne 1982: le premier est un récit de voyage intitulé AAmérique [AAmeryka], qui paraît en 1988, soit cinq années après la mort de l'auteur ; le deuxième est constitué par une partie de son Journal secret [Tajny dziennik], qui n'a été publié qu'en 2012. D'A Amérique, on peut dire qu'il s'agit d'un «anti-journal ». Pour mieux appréhender la particularité de celui-ci, il est intéressant de confronter l'image du Nouveau Monde qui s'en dégage de celle qu'on trouve dans un autre « anti-journal », Amérique, de Jean Baudrillard, publié en 1986. Białoszewski contemple l’Amérique d'un regard, peut-être non dénué de tout a priori, mais façonné par une expérience historique et une vision du monde qui lui sont propres. Baudrillard trouve dans ce voyage américain la confirmation de ses diagnostics catastrophistes.

Mots-clefs : Miron Białoszewski, Jean Baudrillard, journal de voyage, récit de voyage, voyage aux États-Unis.

Miron Biatoszewski was a Polish poet, novelist and playwright. He left two narratives of his trip to the United States in the fall of 1982: the first one is a travel log entitled AAmeryka [Aamerica] published in 1988, five years after Biatoszewski's death: the second one is a part of his Tajny Dziennik [Secret diary], published in 2012. One could say about America, that it is an "anti-diary." We can better appreciate the uniqueness of this book, if we compare his depiction of the New World, with what we can find in America [Amérique], antoher "anti-diary," written by Jean Baudrillard and published in 1986. Biatoszewski contemplates America, maybe with some preconceived ideas, but he is shaped by his own historical experience and worldview. In his American trip, Baudrillard finds, above all, a confirmation of his catastrophist predictions.

Keywords: Miron Biatoszewski, Jean Baudrillard, Travel Diary, Travel Story, trip to the United States. 